\title{
QCD Analysis of the Combined HERA Inclusive Data together with HERA Jet and Charm Data
}

\author{
Gerhard Brandt*i \\ Universität Göttingen \\ E-mail: gbrandtecern. ch
}

\begin{abstract}
This talk presents results of the latest combination of inclusive deep inelastic cross sections published by the H1 and ZEUS collaborations at HERA for neutral and charged current $e^{ \pm} p$ scattering for zero beam polarisation, including charm and jet data. The combined cross sections were used as input to QCD analyses at leading order and next-to-leading order providing a new set of parton distribution functions (PDFs), called HERAPDF2.0Jets. This set is a variation in the HERAPDF2.0 family set of pdfs which is based on fitting DGLAP evolution equations to HERA data only. The inclusion of charm- and jet-production cross sections made a simultaneous determination of these parton distributions and the strong coupling constant possible, resulting in $\alpha_{s}\left(M_{Z}^{2}\right)=$ $0.1183 \pm 0.0009(\exp ) \pm 0.0005$ (model/parameterisation) \pm 0.0012 (hadronisation) ${ }_{-0.0030}^{+0.0037}$ (scale) . Comparisons of cross sections predictions based on HERAPDF2.0Jets NLO to the input data are excellent.
\end{abstract}

The XXIII International Workshop on Deep Inelastic Scattering and Related Subjects

April 27 - May 1, 2015

Southern Methodist University

Dallas, Texas 75275

\footnotetext{
* Speaker.

†n behalf of the H1 and ZEUS collaborations.
} 


\section{Introduction}

Deep inelastic scattering (DIS) of electrons on protons at centre-of-mass energies of up to $\sqrt{s} \simeq 320 \mathrm{GeV}$ at HERA has been central to the exploration of proton structure and quark-gluon dynamics as described by perturbative quantum chromodynamics (pQCD) [1]. The H1 and ZEUS collaborations have recently published a comprehensive QCD analysis of inclusive and various other previously published data [2]. The data were taken at proton beam energies of 920, 820, 575 and $460 \mathrm{GeV}$ and an electron (or positron) beam energy of $27.5 \mathrm{GeV}$. They correspond to an integrated luminosity of about $1 \mathrm{fb}^{-1}$ and span six orders of magnitude in negative four-momentumtransfer squared, $Q^{2}$, and Bjorken $x$.

The name HERAPDF stands for the family of $\mathrm{pQCD}$ analyses based on the DGLAP formalism using only these data. The nominal HERAPDF2.0 pdf set, based only on the inclusive cross sections of the full HERA data set, is described in Ref. [2]. The different variations of HERAPDF2.0 include different assumptions on $Q_{\min }^{2}$, heavy flavour scheme, NLO and NNLO calculations and the inclusion of additional data sets. Both $\mathrm{H} 1$ and ZEUS also published charm production cross sections, some of which were combined and analysed previously [3], and jet production cross sections [4-8]. These data were included in the QCD analysis to obtain the variant HERAPDF2.0Jets, presented in this talk.

\section{Adding HERA Charm Data to the HERAPDF2.0 Fit}

At HERA charm is dominantly produced in virtual photon-gluon fusion. The charm mass parameter $M_{c}$ is an important scale in $\mathrm{pQCD}$ calculations of the process. Sensitivity to the gluon PDF is expected since charm contributes up to $30 \%$ at high $Q^{2}$. However, in a previous QCD analysis using only HERA-I data (HERA data taken in the years up to 2000) the impact of adding charm data to inclusive data as input to NLO QCD fits has been established to mainly result in a reduction of the uncertainty on the charm mass parameter $M_{c}$ for a given flavour scheme [3]. It was also established that the optimal value of $M_{c}$ can differ depending on the choice of a particular general-mass variable-flavour-number scheme (GM-VFNS) chosen for the fit. The fits for all schemes considered were found to be of similar quality in describing the HERA data. For the HERAPDF2.0 analysis, a total of 47 data points on charm production with $Q^{2}$ larger than $Q_{\min }^{2}=3.5 \mathrm{GeV}^{2}$ were added as input to the NLO fits. The 42 sources of correlated systematic uncertainty from the H1 and ZEUS data sets on charm production and two additional sources due to the combination procedure were taken into account. Figure 1 shows $\chi^{2}$ scans of NLO and NNLO pQCD fits to HERA inclusive and combined charm data to determine the optimal value of $M_{c}$. The GM-VFNS RTOPT flavor scheme was used in the fits. The optimal value $M_{c}=1.47 \mathrm{GeV}$ at NLO $\left(M_{c}=1.43 \mathrm{GeV}\right.$ at NNLO) are chosen at minimum $\chi^{2}$ and are used in subsequent fits. Fits including the charm data were proven to be consistent with fits using the inclusive data only, but only a marginal reduction in the uncertainty on the low-x gluon PDF was obtained.

\section{Adding HERA Jet Data to the HERAPDF2.0fit}

In pQCD fits to inclusive DIS data alone, the gluon PDF is determined indirectly via the DGLAP equations using the observed scaling violations, while the value of $\alpha_{s}\left(M_{Z}^{2}\right)$ is given as 


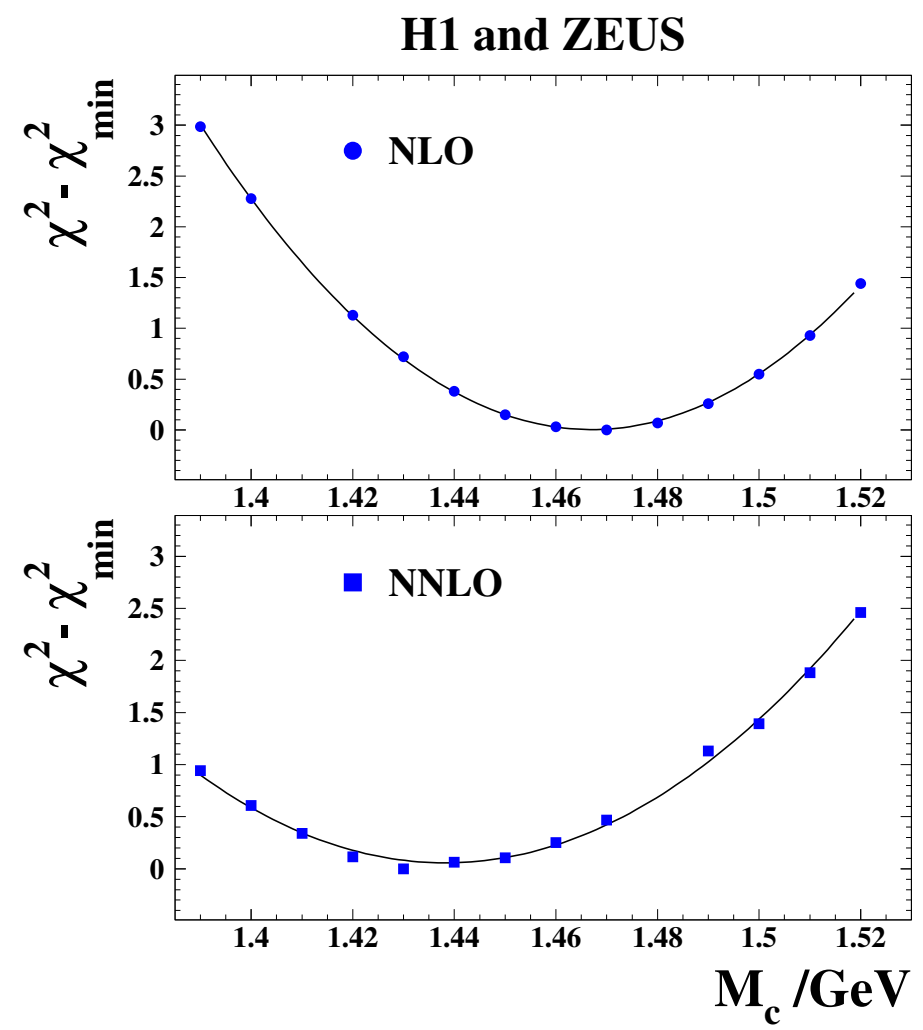

Figure 1: The $\Delta \chi^{2}=\chi^{2}-\chi_{\min }^{2}$ versus the charm mass parameter $M_{c}$ for NLO and NNLO fits based on the combined data on charm production in addition to the combined inclusive data.

input from external information [9]. Data on jet production cross sections provide an independent measurement of the gluon distribution. They are sensitive to the gluon distribution at lower $Q^{2}$ and to the valence-quark distribution at higher $Q^{2}$. The inclusion of jet data in $\mathrm{pQCD}$ fits not only reduces the uncertainty on the high-x gluon distribution in fits with fixed $\alpha_{s}\left(M_{Z}^{2}\right)$ but also allow the accurate simultaneous determination of $\alpha_{s}\left(M_{Z}^{2}\right)$ and the gluon distribution. This is demonstrated in Fig. 2 where a $\chi^{2}$ fit to $\alpha_{s}\left(M_{Z}^{2}\right)$ is shown. Fits including these jet data and including the combined charm data were performed with $\alpha_{s}\left(M_{Z}^{2}\right)=0.118$ fixed and with $\alpha_{s}\left(M_{Z}^{2}\right)$ as a free parameter in the fit. This inclusion was first used to validate the choice of $\alpha_{s}\left(M_{Z}^{2}\right)=0.118$ for HERAPDF2.0 by investigating the dependence of the $\chi^{2}$ s of the HERAPDF2.0 pQCD fits on $\alpha_{s}\left(M_{Z}^{2}\right)$. Three $\chi^{2}$ scans vs. the value of $\alpha_{s}\left(M_{Z}^{2}\right)$ were performed at NLO for three values of $Q_{\min }^{2}$. The result is depicted in the top panel of Fig. 2. A distinct minimum at $\alpha_{s}\left(M_{Z}^{2}\right) \approx 0.118$ is observed, which is basically independent of $Q_{\min }^{2}$. This validates the choice of $\alpha_{s}\left(M_{Z}^{2}\right)=0.118$ for HERAPDF2.0 NLO. Scans at NLO and NNLO were also performed for fits to inclusive data only. The middle and bottom panels of Fig. 2 show that these scans yielded similar shallow $\chi^{2}$ dependences and the minima were strongly dependent on the $Q_{\min }^{2}$. This demonstrates that the inclusive data alone cannot constrain $\alpha_{s}\left(M_{Z}^{2}\right)$ reasonably.

The resulting HERAPDF variant with free $\alpha_{s}\left(M_{Z}^{2}\right)$ is called HERAPDF2.0Jets. A full uncertainty analysis was performed for the HERAPDF2.0Jets variant, including model and parameterisation uncertainties and additional hadronisation uncertainties on the jet data as evaluated for the 
a)
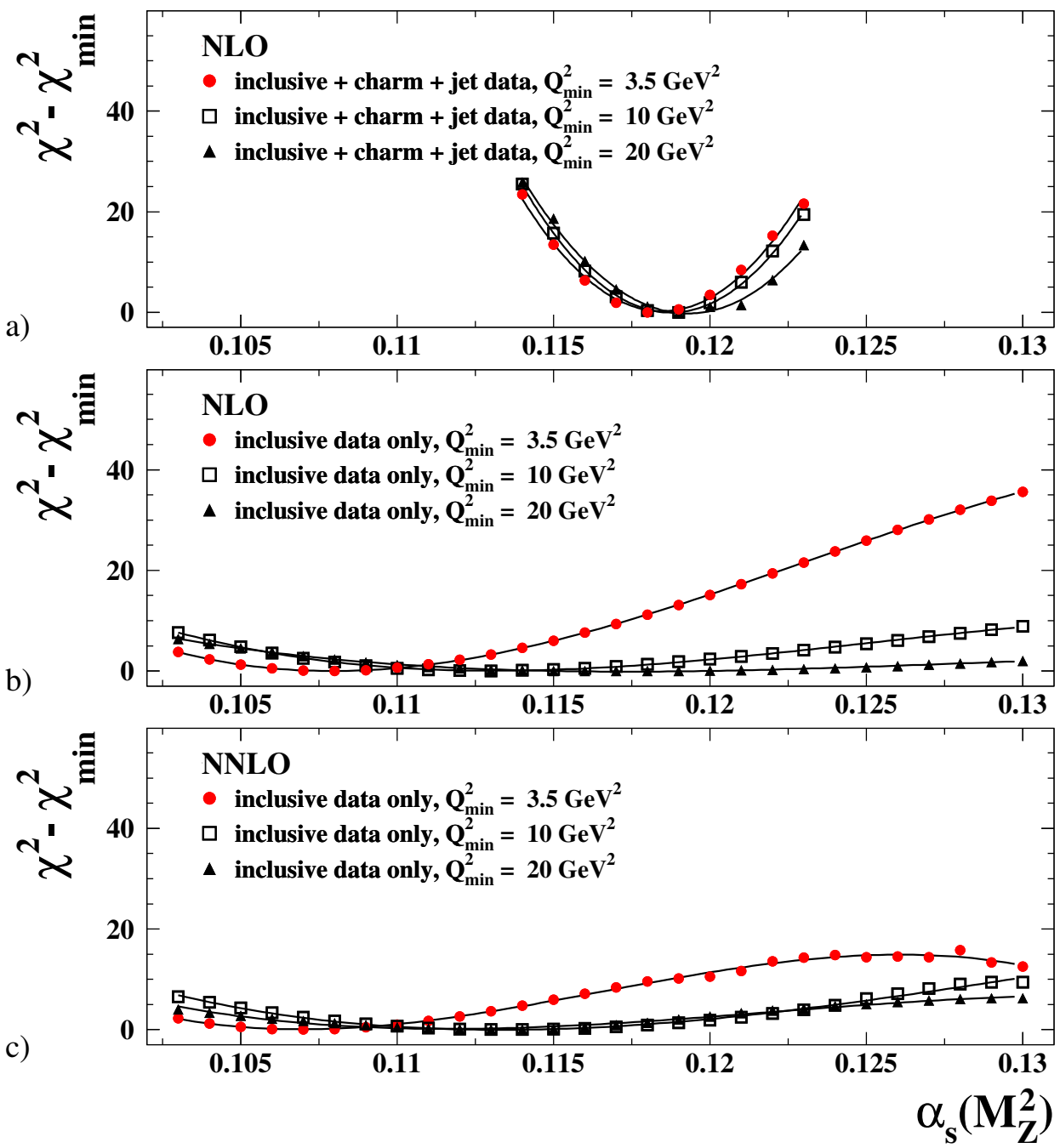

Figure 2: $\Delta \chi^{2}=\chi^{2}-\chi_{\min }^{2}$ vs. $\alpha_{s}\left(M_{Z}^{2}\right)$ for pQCD fits with different $Q_{\min }^{2}$ using data on (a) inclusive, charm and jet production at NLO, (b) inclusive $e p$ scattering only at NLO and (c) inclusive $e p$ scattering only at NNLO.

original publications [4-8].

The PDFs resulting from a fit with free $\alpha_{S}\left(M_{Z}^{2}\right)$, HERAPDF2.0Jets, and from a fit with fixed $\alpha_{s}\left(M_{Z}^{2}\right)=0.118$ are shown in Fig. 3. A full uncertainty analysis was performed in both cases, including model and parameterisation uncertainties as well as additional hadronisation uncertainties on the jet data. The PDFs are very similar, because the HERAPDF2.0Jets fit with free $\alpha_{s}\left(M_{Z}^{2}\right)$ yields a value which is very close to the value used for the fit with fixed $\alpha_{s}\left(M_{Z}^{2}\right)$. The jet data determine the value of $\alpha_{s}\left(M_{Z}^{2}\right)$ very well in the HERAPDF2.0Jets fit. Thus, the uncertainty on $\alpha_{s}\left(M_{Z}^{2}\right)$ in this fit does not significantly increase the uncertainty on the gluon PDF with respect to the fit with $\alpha_{s}\left(M_{Z}^{2}\right)$ fixed. The difference in the $\alpha_{s}\left(M_{Z}^{2}\right)$ free fit is mostly due to extra uncertainty coming from the hadronisation corrections.

The PDFs from the HERAPDF2.0Jets fit with $\alpha_{s}\left(M_{Z}^{2}\right)=0.118$ fixed are also very similar to the standard PDFs from HERAPDF2.0 NLO. This is demonstrated in Fig. 4. This is again the 
result of the choice of $\alpha_{s}\left(M_{Z}^{2}\right)=0.118$ for HERAPDF2.0 which is also the preferred value for HERAPDF2.0Jets. Consequently, there is only a small reduction of the uncertainty on the gluon distribution observed for HERAPDF2.0Jets.

The $\chi^{2}$ of the HERAPDF2.0Jets fit with free $\alpha_{s}\left(M_{Z}^{2}\right)$ is the same as for the fit with fixed $\alpha_{s}\left(M_{Z}^{2}\right)=0.118$. This is again due the fact that the value of $\alpha_{s}\left(M_{Z}^{2}\right)$ obtained from the fit is very close to the value previously fixed. The strong coupling constant obtained is

$$
\begin{array}{r}
\alpha_{s}\left(M_{Z}^{2}\right)=0.1183 \pm 0.0009(\exp ) \pm 0.0005(\text { model } / \text { parameterisation }) \\
\pm 0.0012(\text { hadronisation }){ }_{-0.0030}^{+0.0037}(\text { scale })
\end{array}
$$

The uncertainty on $\alpha_{s}\left(M_{Z}^{2}\right)$ due to scale uncertainties was evaluated by varying the renormalisation and factorisation scales by a factor of two, both separately and simultaneously, and taking the maximal positive and negative deviations. The uncertainties were assumed to be $50 \%$ correlated and $50 \%$ uncorrelated between bins and data sets. This resulted in an asymmetric uncertainty of +0.0037 and -0.0030 . The result on $\alpha_{s}\left(M_{Z}^{2}\right)$ is compatible with the world average [9] and it is competitive with other determinations at NLO.

\subsection{Comparison of HERAPDF2.0Jets NLO to data}

The predictions of HERAPDF2.0Jets NLO with free $\alpha_{s}\left(M_{Z}^{2}\right)$ were compared to charm input data [3] and the description of the data is excellent. Comparisons of the predictions of HERAPDF2.0Jets to the data on jet production used as input [4-8]. were also done. All analyses were performed using the assumption of massless jets, i.e. the transverse energy, $E_{T}$, and the transverse momentum of a jet, $p_{T}$, are equivalent. For inclusive jet analyses, each jet is entered separately with its $p_{T}$. For dijet and trijet analyses, the average of the transverse momenta is used as $p_{T}$. These different definitions of $p_{T}$ were also used to set the the renormalisation scale to $\mu_{\mathrm{r}}^{2}=\left(Q^{2}+p_{T}^{2}\right) / 2$ for calculating predictions. The factorisation scale was chosen as $\mu_{\mathrm{f}}^{2}=Q^{2}$. Scale uncertainties were not considered for the comparisons to data. The description of all the data on jet production by HERAPDF2.0Jets NLO is also excellent.

\section{References}

[1] A. Cooper-Sarkar and R. Devenish, Deep inelastic Scattering, Oxford Univ. Press (2011), ISBN 978-0-19-960225-4.

[2] H. Abramowicz et al., [H1 and ZEUS Collaborations], [arXiv:1506.06042 [hep-ex]].

[3] F. Aaron et al., [H1 and ZEUS Collaborations], Eur. Phys. J. C 73, 2311 (2013), [arXiv:1211.1182].

[4] S. Chekanov et al. [ZEUS Collaboration], Phys. Lett. B 547, 164 (2002), [hep-ex/0208037].

[5] H. Abramowicz et al. [ZEUS Collaboration], Eur. Phys. J. C 70, 965 (2010), [arXiv:1010.6167].

[6] F. Aaron et al. [H1 Collaboration], Eur. Phys. J. C 67, 1 (2010), [arXiv:0911.5678]. 

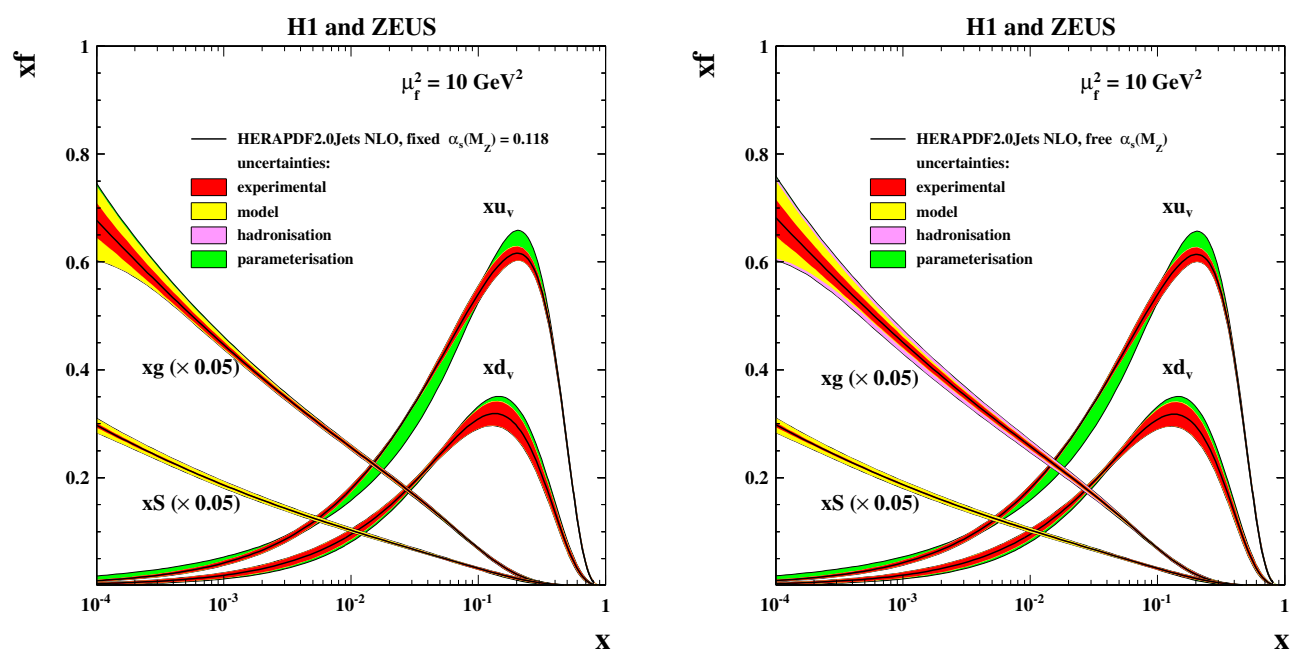

Figure 3: The parton distribution functions $x u_{v}, x d_{v}, x S=2 x(\bar{U}+\bar{D})$ and $x g$ of HERAPDF2.0Jets NLO at $\mu_{\mathrm{f}}^{2}=10 \mathrm{GeV}^{2}$ with fixed $\alpha_{s}\left(M_{Z}^{2}\right)=0.118$ (top) and free $\alpha_{s}\left(M_{Z}^{2}\right)$ (bottom). The experimental, model and parameterisation uncertainties are shown. The hadronisation uncertainty is also included, but it is only visible for the fit with free $\alpha_{\mathrm{s}}\left(M_{7}^{2}\right)$.
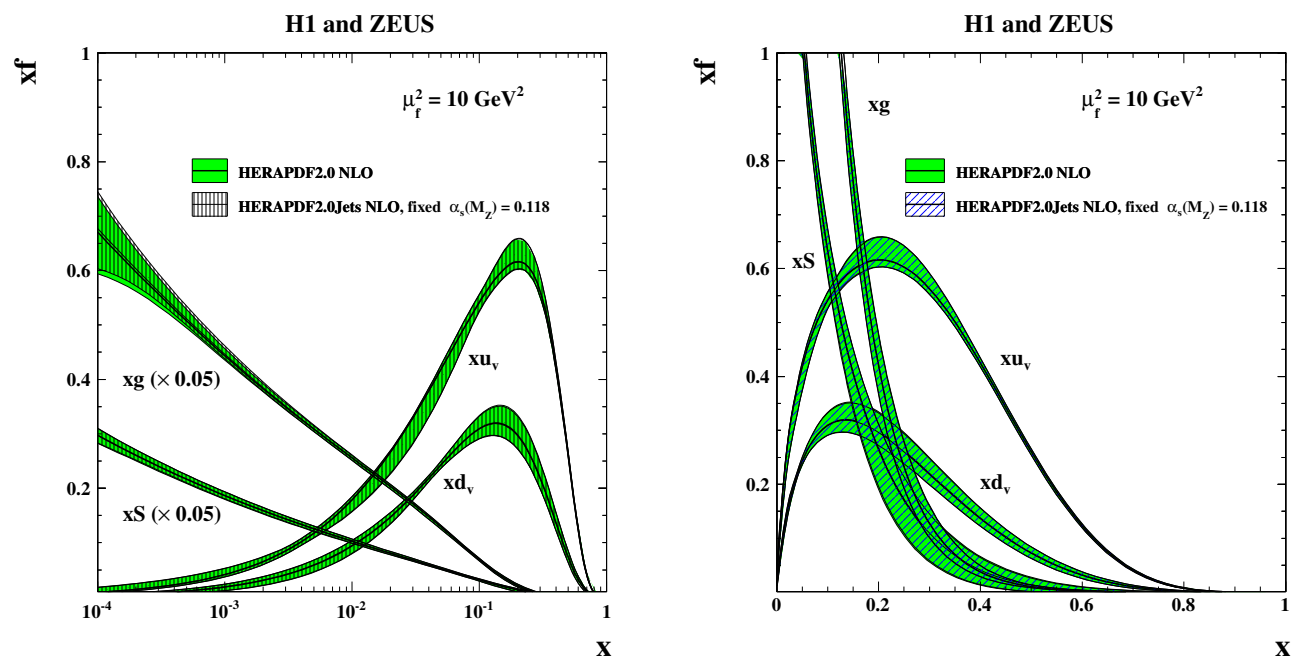

Figure 4: The parton distribution functions $x u_{v}, x d_{v}, x S=2 x(\bar{U}+\bar{D})$ and $x g$ of HERAPDF2.0Jets NLO at $\mu_{\mathrm{f}}^{2}=10 \mathrm{GeV}^{2}$ compared to those of HERAPDF2.0 NLO on logarithmic (top) and linear (bottom) scales. The fits were done with fixed $\alpha_{s}\left(M_{Z}^{2}\right)=0.118$. The bands represent the total uncertainties.

[7] A. Aktas et al. [H1 Collaboration], Phys. Lett. B 653, 134 (2007), [arXiv:0706.3722].

[8] V. Andreev et al. [H1 Collaboration], Eur. Phys. J. C 65, 2 (2015), [arXiv:1406.4709].

[9] J. Beringer et al. (Particle Data Group), Phys. Rev. D 86, 010001 (2012). 